\title{
RF Wafer Probing with Improved Contact Repeatability Using Nanometer Positioning
}

\author{
Khadim Daffé 1 , Gilles Dambrine ${ }^{1}$, Fabian von Kleist-Retzow ${ }^{2}$, Kamel Haddadi ${ }^{1}$ \\ ${ }^{1}$ Institute of Electronics, Microelectronics and Nanotechnology (IEMN-CNRS), University of Lille, Villeneuve d'Ascq, France \\ ${ }^{2}$ Division Microrobotics and Control Engineering, University of Oldenburg, Oldenburg, Germany
}

\begin{abstract}
This paper presents an improved technique for monitoring and controlling the contact condition of on-wafer RF probes with nanometer accuracy to enhance the measurement repeatability. The set-up consists of a vector network analyzer, a modified probe station with a planar calibration substrate aligned under microwave GSG probe through a closed-loop nanopositioner and a camera system. A fully one-port SOL calibration is performed in the frequency range 0.05-50 GHz. A repeatability study based on standard deviations of the measured data considering both conventional and proposed approaches is described. From these experimental results, the improvement of the technique proposed is achieved by accurately controlling the probe contacts.
\end{abstract}

Index Terms - On-wafer measurements, one-port measurement, calibration, short-open-load (SOL), vector network analyzer (VNA), ground-signal-ground (GSG) probe, automation.

\section{Context And Motivations}

The motivation of this study is driven by the need to characterize microwave planar devices beyond the capability of the existing commercially available measurement solutions. In particular, the electrical characterization of high impedance nanodevices like nanotubes, nanowires, self-assembled molecular monolayers, atoFarad capacitances is a well-known scientific challenge [1]-[3]. The main limitation is the lack of measurement sensitivity of the VNA that corresponds to reflection coefficients of high impedance devices close to one [4]. Therefore, one of the main issues is to ensure the contact probe repeatability for both calibration process and device under test (DUT) measurement to preserve the measurement resolution.

A typical RF device characterization set-up consists of a vector network analyzer (VNA), a probe station with a pair of microwave GSG probes aligned manually or automatically through a microscope or a camera system onto calibration substrate and test devices. The RF measurement is enhanced by calibrating the measurement set-up at DUT interface. A variety of error models and related calibration procedures have been described in the literature [5]-[13]. Although these models have been successfully applied up to the $\mathrm{THz}$ range [14], stochastic errors such as drift, stability, and contact repeatability degrade the measurement accuracy, especially as the frequency is raised. For this latter, $S$-parameters measurements are known to be very sensitive to $\mathrm{X}, \mathrm{Y}, \mathrm{Z}$, and $\Theta$ positioning of the probe contacts with respect to calibration standards, dummy structures and devices pads. Semiautomated or fully automated probe system exhibits at the best $0.2 \mu \mathrm{m}$ resolution in $\mathrm{X}, \mathrm{Y}, \mathrm{Z}$ and $\Theta$ and repeatability in the order of the micron. For comparison, it remains at least three orders of magnitude higher than the mechanical accuracy $(\cong 1$ $\mathrm{nm}$ ) of a scanning probe microscope (SPM) using close-loop piezo-based positioners [15]-[18].

The main objective of the work is to evaluate the capabilities of piezo-based positioning methods to monitor and control the contact behavior between the probe and the DUT. To that end, reflection coefficients measured on SOL calibration loads are measured in the frequency range $0.05-50 \mathrm{GHz}$ by both a manual positioner (Z-axis: $\pm 2.5 \mu \mathrm{m}$ ) and a piezoelectric based nano-positioner (Z-axis: $\pm 25 \mathrm{~nm}$ ). The experimental set-up and measurement procedure are depicted in Section II. In Section III, discussion is proposed on the basis of standard deviations calculated from uncalibrated and calibrated measured data. In particular, frequency signatures of the different stochastic errors sources (mechanical repeatability, drift, stability) are highlighted.

\section{A. Description of the set-up}

The set-up is depicted in Fig. 1.

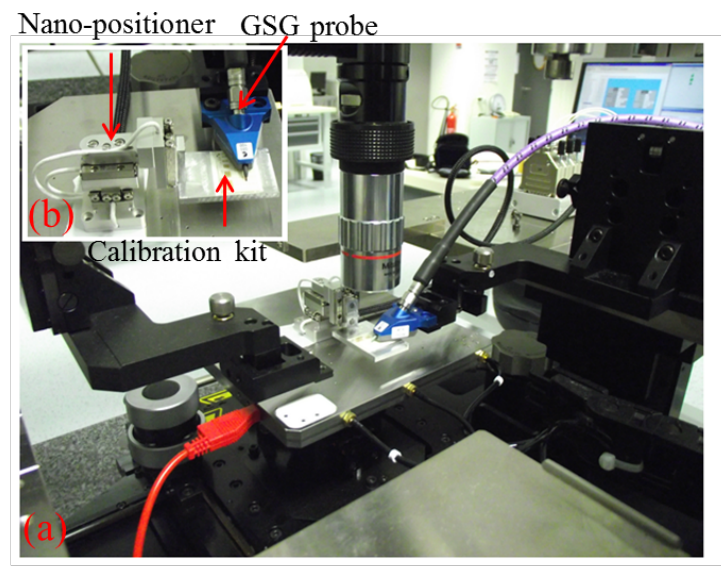

Fig. 1. (a) Modified Wafer probe station for on-wafer measurements in the range 0.05-50 GHz. (b) Mounting of the sample holder on the nano-positioning stage. 
An N5225A PNA VNA from ${ }^{\circledR}$ Keysight is used to measure the reflection coefficients. The frequency step is set to $100 \mathrm{MHz}$ (491 points) in the frequency range spanning from $50 \mathrm{MHz}$ to $50 \mathrm{GHz}$. The RF signal power is set to $0 \mathrm{dBm}$ and an intermediate frequency bandwidth (IFBW) of $100 \mathrm{~Hz}$ is used. The positioner is equipped with a $100 \mu \mathrm{m}$ pitch GSG ®Infinity probe. A microwave cable $(2.4 \mathrm{~mm})$ from ${ }^{\circledR}$ Gore with high phase and amplitude stability with flexure is used to connect the probe to the VNA. The Impedance Standard Substrate (ISS) reference is \#101-190 from ${ }^{\circledR}$ Cascade Microtech. The mechanical on-wafer station is a MPS150 from ${ }^{\circledR}$ Cascade with $\mathrm{XYZ}$ positioner having an estimated positioning resolution of $+/-2.5 \mu \mathrm{m}$ which corresponds to half of the minimum cursor rule [Fig. 1(a)].

The reference of the nano-positioning stage [see Fig. 1(b)] is XYZ_SLC1720s_SBP from ${ }^{\circledR}$ SmarAct $\mathrm{GmbH}$. The system is based on three tool $\operatorname{arms}(\mathrm{X}, \mathrm{Y}$ and $\mathrm{Z})$ that provide fine positioning capabilities (resolution $=1 \mathrm{~nm}$; repeatability $=25$ $\mathrm{nm}$ ) based on piezo-electric slip-stick axes equipped with nanosensors. The positioners are controlled by a PC via an interface box. The station involves $(5 \mathrm{x} / 0.14)$ zoom optical system equipped by a video camera.

All measurements are carried out in a controlled environment (IEMN Nano-characterization center) with temperature variations less than $+/-1^{\circ}$, anti-vibration building and experimental tables as well as stable ambient hygrometry close to $50 \%$.

\section{B. Methodology}

First we have to mention that the measurements were performed during two consecutive half days, devoted respectively for manual positioning and for piezoelectric nano-positioning. The ISS is fixed to a mechanical part of the piezo-electric positioner, itself fixed on the sample holder of the station (see Fig. 1). On the ISS, a set of three contact standards corresponding to one-port SOL calibration is considered. For both cases, 15 measurements per load are performed to derive statistical information. To provide a reference measurement independent of the probe contact repeatability, an open-air 'standard' is considered (probe in free-space conditions). The definitions of the standards, corresponding to those provided by the ISS supplier, are fixed once for all. The total amount of one-port data (magnitude and phase of $S_{11}$ ) versus frequency is of 117840 . The main idea of the measurement protocol was to move only the Z-axis position both in manual and piezo-electric systems. ISS alignment and $\mathrm{XYZ} \Theta$ probes adjustments, to ensure repeatable contacts, were done first and once for all. The standards were moved successively by using the XY axis of the piezoelectric nano-positioner to ensure a good positioning repeatability and same high resolution for both manual and piezoelectric measurement campaigns. For manual positioning the Z-axis is moved up and down to the same cursor position for all measurements; for piezoelectric nano-positioner the Z-axis was controlled in close loop operation.

\section{RESULTS AND DISCUSSIONS}

To investigate the measurement performance in terms of measurement accuracy and repeatability, mean and standard deviations $(2 \sigma)$ of the complex reflection coefficients $S_{11}$ are determined.

The mean of the reflection coefficient is given by

$$
\overline{\mathrm{S}_{11}}=\frac{1}{n} \sum_{i=1}^{n} S_{1 i_{i}}
$$

with $n=15$, number of measurements.

The standard deviation of the complex reflection coefficient is defined by

$$
\sigma=\left(\frac{1}{n-1} \sum_{i=1}^{n}\left|S_{11_{i}}-\overline{S_{11}}\right|^{2}\right)^{\frac{1}{2}}
$$

The standard deviations $(2 \sigma)$ of the corresponding data are plotted in Fig. 2. Concerning the 'short' standard [see Fig. 2 (a)] that is the most reflective one ('open' standard is not a contacted device), the standard deviation is reduced by a factor 4 when the nano-positioning method is considered up to $20 \mathrm{GHz}$. Therefore, in this frequency range, the accurate control of Z-position is strongly influent. As intermediary conclusion, up to $10-20 \mathrm{GHz}$ is certainly the main interesting range for nano-devices measurements; we proof here that part of uncertainties can be better controlled in this frequency range. Moreover, this frequency range is enough broad (many decades) to perform accurate characterization of sub-fF capacitances or/and above $10 \mathrm{k} \Omega$ resistances.

From 20 to $50 \mathrm{GHz}$, the benefit is less significant. Other sources of uncertainties become predominant. The total campaign duration was close to one day; instrumental drift, whose influence increases with frequency of operation, becomes the predominant source of uncertainties. This drift over time effect is qualitatively shown in the same figure [Fig. 2 (a)] by reporting the 'standard deviations' observed for four consecutive measurements (piezo-electric case).

As predicted, the open-air standard deviations depicted in Fig. 2 (b) are not influenced by this experimental comparison. The same advantage using a nano-positioner is obtained on the reflection coefficient of the 'load' standard up to $20 \mathrm{GHz}$. The standard deviation of the reflection coefficient $S_{11}$ is reduced up to $20 \mathrm{GHz}$.

To better appreciate the measurement accuracy, the mean relative errors on the measured reflection coefficients for the short and match loads are determined (TABLE 1). 
TABLE I RELATIVE ERRORS ON THE MEASURED REFLECTION COEFFICIENTS.

\begin{tabular}{|c|c|c|c|c|}
\cline { 2 - 5 } \multicolumn{1}{c|}{} & \multicolumn{2}{c|}{$0.05-20 \mathrm{GHz}$} & \multicolumn{2}{c|}{$20-50 \mathrm{GHz}$} \\
\cline { 2 - 5 } \multicolumn{1}{c|}{} & manual & piezo & manual & piezo \\
\hline SHORT & $0.23 \%$ & $0.06 \%$ & $0.44 \%$ & $0.18 \%$ \\
\hline MATCH & $0.35 \%$ & $0.1 \%$ & $0.42 \%$ & $0.16 \%$ \\
\hline
\end{tabular}

From TABLE 1, when considering the frequency range $0.05-20 \mathrm{GHz}$, the measurement repeatability is increased by a factor around 3.5 for both short and match loads. For the upper frequency range, the improvement is lower (factor 2.5 ). It is also noticed that these relative errors are nearly equal for the two loads considered in the range 20-50 GHz. This degradation of the measurement repeatability is therefore imputed to drift error.
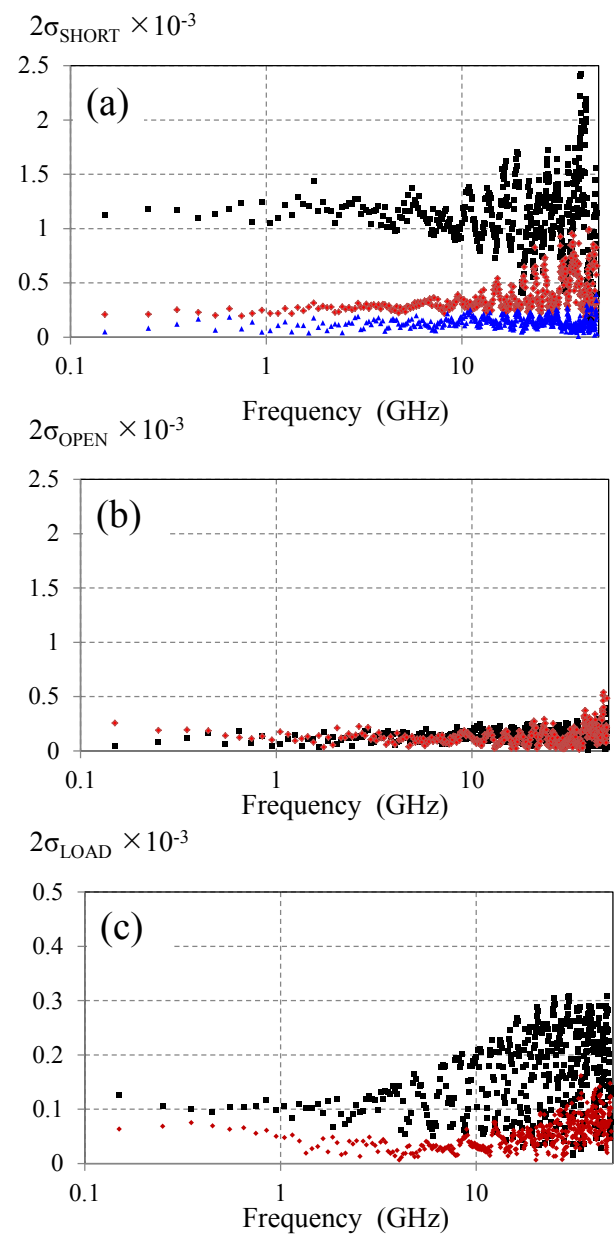

Fig. 2. Standard deviations of the reflecion coefficient $S_{11}$ as a function of the frequency (loads are respectively short, open and load ISS standards ) determined for $n$ consecutive measurements (piezo-electric positioning : $n=15$ and $\boldsymbol{\Delta} n=4$, mechanical positioning : $\mathbf{\square} n=15$ ).

\section{CONCLUSION}

The study performed in this article reports a significant performance improvement of contact repeatability in RF wafer probing. In particular, conventional on-wafer probing system based on mechanical displacements has been compared to a home-made nano-positioning platform. Using the same measurement configurations, it has been shown that the contact repeatability can be readily improved in the microwave range up to $20 \mathrm{GHz}$. Furthermore, other stochastic errors such as drift become predominant in the range 20-50 GHz. These results are instructive and beneficial to further experiments. Furthermore, future works include the automation of the probing process to reduce the measurement duration time. In particular, a complete study will be devoted to study the impact of the drift (acquisition time) on calibrated $S$-parameters using conventional and the proposed approach.

\section{ACKNOWLEDGEMENT}

This work is performed under Nano2017 (http://www.st.com/web/en/press/c2727) and EMPIR Planarcal projects (http://www.planarcal.ptb.de). This work used the facilities within the EQPX ExCELSiOR project.

\section{REFERENCES}

[1] P. Rice, T. M. Wallis, S. E. Russek, and P. Kabos, "Broadband electrical characterization of multiwalled carbon nanotubes and contacts," Nano Lett., vol. 7, no. 4, pp. 1086-1090, Mars 2007.

[2] L. Nougaret, G. Dambrine, S. Lepilliet, H. Happy, N. Chimot, V. Derycke, and J.-P. Bourgoin, "Gigahertz characterization of a single carbon nanotube," Appl. Phys. Lett., vol. 96, no. 4, pp. 042109-1-042109-3, Jan. 2010.

[3] The International Technology Roadmap for Semiconductors (ITRS), 2013. http://www.itrs.net/Links/2013ITRS/2013Chapters/2013ERD.pdf

[4] H. Happy, K. Haddadi, D. Théron, T. Lasri, and G. Dambrine, "Measurement techniques for RF nanoelectronic devices : new equipment to overcome the problems of impedance and scale mismatch," IEEE Microwave Magazine, vol. 15, no. 1, pp. 30-39. Jan. 2014.

[5] G.F. Engen and C.A. Hoer, "Thru-Reflect-Line: An Improved Technique for Calibrating the Dual Six-Port Automatic Network Analyzer," IEEE Trans. Microw. Theory Tech., vo1.27, pp. 987-993, Dec. 1979.

[6] N.M. Ridler and M.J. Salter, "Evaluating and expressing uncertainty in complex S-parameter measurements," IEEE $56^{\text {th }}$ ARFTG Microw. Meas. Conf, pp.43-75, Nov. 2000.

[7] D. Rytting, "An analysis of vector measurement accuracy enhancement Techniques," RF and Microwave Symp. Exhibition, 1980.

[8] F. Mubarak and G. Rietveld, "Residual error Analysis of a calibrated Vector Network Analyzer," IEEE $84^{\text {th }}$ ARFTG Microw. Meas. Conf, pp $1-6,2014$.

[9] M. Horibe, M. Shida, and K. Komiyama, "VNA Traceability Tool," IEEE 73 ${ }^{\text {st }}$ ARFTG Microw. Meas. Conf, pp 1-6, 2009.

[10] M. Wollensack, J. Hoffmann, J. Ruefenacht, and M. Zeier, "VNA Tools II: S-parameter uncertainty calculation," IEEE $79^{\text {th }}$ ARFTG Microw. Meas. Conf, 2012. 
[11] V. Teppati and A. Ferrero, "A Comparison of Uncertainty Evaluation Methods for On-Wafer -Parameter Measurements," IEEE Instrum. and Meas., vol. 63, no. 4, pp. 935-942, April 2014.

[12] K. Wong, "Traceability of vector network analyzer measurements," $2^{\text {nd }}$ ARFTG Microw. Meas. Conf., pp. 157-167, Dec. 2008.

[13] J. Martens, "LRM: A quantitative look at reference impedance contradictions and other uncertainty impacts," $69^{\text {th }}$ ARFTG Microw. Meas. Conf, pp. 1-7, Jun. 2007.

[14] T. J. Reck, L. Chen, C. Zhang, A. Arsenovic, C. Groppi, A. W. Lichtenberger, R. M. Weikle, II, and N. S. Barker, "Micromachined probes for submillimeter-wave on-wafer measurements-Part II: RF design and characterization," IEEE Trans. Terahertz Sci. Tech., vol. 1, no. 2, pp. 357-363, 2011.

[15] T. Dargent, K. Haddadi, T. Lasri, N. Clément, D. Ducatteau, B. Legrand, H. Tanbakuchi, and D. Théron, "An interferometric scanning microwave microscope and calibration method for sub-fF microwave measurements," Rev. Sci. Instrum., vol. 84, no.12, pp. 123705-123705-7, Dec. 2013.

[16] F. T. von Kleist-Retzow, T. Tiemerding, P. Elfert, O. C. Hänßler, and S. Fatikow, "Automated Calibration of RF On-Wafer Probing and Evaluation of Probe Misalignment Effects using a Desktop MicroFactory," $2^{\text {nd }}$ Conf. Microw. and Terahertz Tech, 2016.

[17] J. Marzouk, S. Arscott, K. Haddadi, T. Lasri, C. Boyaval, S. Lepilliet, and G. Dambrine, "Miniaturized microcantilever-based RF microwave probes using MEMS technologies," Procedia Eng., vol. 87, pp. 692695, 2014.

[18] A. El Fellahi, K. Haddadi, J. Marzouk, S. Arscott, C. Boyaval, T. Lasri, and G. Dambrine, "Integrated MEMS RF Probe for SEM Station-Pad Size and Parasitic Capacitance Reduction," IEEE Microw. Wireless Comp. Lett, vol. 25, no. 10, pp. 693-695, Oct. 2015. 